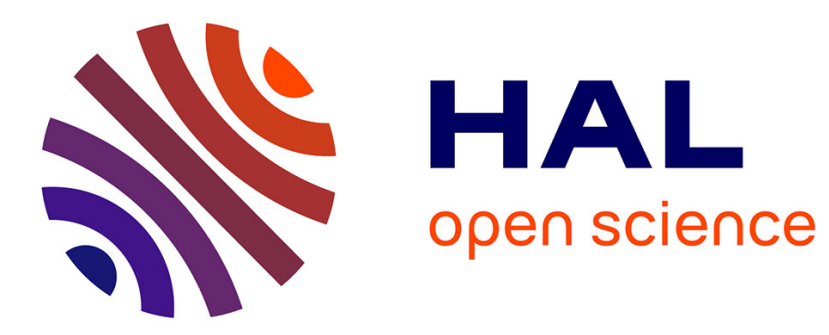

\title{
On stable quantum currents
}

Joachim Asch, Olivier Bourget, Alain Joye

\section{To cite this version:}

Joachim Asch, Olivier Bourget, Alain Joye. On stable quantum currents. Journal of Mathematical Physics, 2020, 61 (9), pp.092104. 10.1063/5.0005737 . hal-02160583v2

\section{HAL Id: hal-02160583 \\ https://hal.science/hal-02160583v2}

Submitted on 8 Oct 2020

HAL is a multi-disciplinary open access archive for the deposit and dissemination of scientific research documents, whether they are published or not. The documents may come from teaching and research institutions in France or abroad, or from public or private research centers.
L'archive ouverte pluridisciplinaire HAL, est destinée au dépôt et à la diffusion de documents scientifiques de niveau recherche, publiés ou non, émanant des établissements d'enseignement et de recherche français ou étrangers, des laboratoires publics ou privés. 


\title{
On stable quantum currents $* \dagger$
}

\author{
Joachim Asch $\ddagger$ Olivier Bourget $\S$ Alain Joye $₫$ \\ $21 / 02 / 2020$
}

\begin{abstract}
We study transport properties of discrete quantum dynamical systems on the lattice, in particular Coined Quantum Walks and the Chalker-Coddington model. We prove existence of a non trivial charge transport implying that the absolutely continuous spectrum covers the whole unit circle under mild assumptions. We discuss anomalous quantum charge transport. For Quantum Walks we exhibit explicit constructions of coins which imply existence of stable directed quantum currents along classical curves. The results are of topological nature and independent of the details of the model.
\end{abstract}

\section{Introduction}

We consider the signature of transport provided by the presence of a non trivial absolutely continuous component in the spectrum of the evolution operator of two classes of unitary network models: the celebrated Chalker-Coddington model of condensed matter physics, $[11,19]$, which provides an effective description of one time step of the motion of an electron in a plane subject to a strong perpendicular magnetic field and a random potential, and the abstract $d$-dimensional Coined Quantum Walk which finds applications in several areas of Quantum Computing, [25, 21]. Both models are characterized by a unitary operator

$$
U \text { on a Hilbert space } \ell^{2}\left(\mathbb{Z}^{d}, \mathbb{C}^{n}\right)
$$

\footnotetext{
*Supported by FONDECYT 1161732, and ECOS-Conicyt C15E10

†Supported by the LabEx PERSYVAL-Lab (ANR-11- LABEX- 0025-01) funded by the French program Investissement d'Avenir and ANR Grant NONSTOPS (ANR-17-CE40-0006-01)

${ }_{\ddagger}^{\ddagger}$ CNRS, CPT, Aix Marseille Université, Université de Toulon, Marseille, France, asch@cpt.univmrs.fr

$\S$ Departamento de Matemáticas Pontificia Universidad Católica de Chile, Av. Vicuña Mackenna 4860, C.P. 69044 11, Macul Santiago, Chile

IUniversité Grenoble Alpes, CNRS Institut Fourier, 38000 Grenoble, France
} 
which couples neighboring sites only.

Moreover both classes are parametrised by a countable family of unitary finitedimensional matrices that describe the local dynamics: the scattering matrices for the Chalker-Coddington model and the coin matrices for the Quantum Walks. This makes these models very versatile and they display a whole range of quantum dynamics depending on the choice of these matrices. In particular, it is known that dynamical Anderson localisation takes place for random versions of the Chalker-Coddington model, $[1,2]$, and of Coined Quantum Walks, $[16,6,17]$. On the other hand for Quantum Walks on trees there are localisation-delocalisation transitions, [14] and the localisation length diverges with high coordination number for Balanced Random Quantum Walks, [5]. Moreover, homogeneous parameters at infinity or the presence of boundaries induce absolutely continuous spectrum for both models, $[15,3,4]$. For periodically driven unitary networks models it is known that the presence of boundaries and symmetries of the bulk imply occurence of currents, $[22,12,13,24]$.

By contrast, here we analyse transport properties of static models defined on the whole of $\mathbb{Z}^{d}$.

To do so we use topological properties of the self-adjoint flux operator out of a subspace $\operatorname{Ran}(P)$ of an orthogonal projection

$$
\Phi=U^{*} P U-P .
$$

The index of $\Phi$ is defined by the Fredholm index of the restriction of $U$ to $\operatorname{Ran}(P)$, see (1) and proposition 2.2 below. It is an instance of the index of a pair of projections which is invariant under small or compact variations of $U$ or $P$. A trace formula in odd powers of $\Phi$ is known for the index which is of essential importance for the theory of the quantum Hall effect $[7,8]$. We prove in general that a non trivial index implies the appearance of a wandering subspace, reducing a perturbation of the evolution operator which becomes a shift on the subspace. This shift may be considered as a current. We prove that under regularity assumptions it implies the occurence of stable gapless absolutely continuous spectrum, see Theorem 2.1 . In the sequel we provide relevant physical examples where these assumptions hold and also examples where the current is anomalous, existing in a fully localised system.

We observe that a trace formula in even powers of $\Phi$ holds for its index which generalises Kitaev's formula [18], see Proposition 2.2.

Then we turn to Coined Quantum Walks. For $P$ being the fully filled state on a half space we analyse the properties of $\Phi$ as a function of the local coin matrices. We show how to engineer currents along leads on an interface by choosing coin matrices, Theorem 3.16. These currents imply existence of absolutely continuous spectrum stable under perturbations at all quasienergies.

The fourth Section is devoted to the Chalker-Coddington model defined on the plane. We show that under very general conditions there exists a non trivial flux of 
particles transversal to a curve. Under mild additional assumptions this implies gapless absolutely continuous spectrum, see Theorem 4.7.

In the last section we conclude with remarks on anomalous charge transport.

\section{General results}

In [7] the relative index of two projections was defined and shown to be zero if and only if there exists a unitary operator interchanging the projections; also a trace formula in odd powers of their difference was shown for the index.

In this section we shall prove in general that stable currents and spectral properties of $U$ are implied by a non vanishing relative index of $\Phi$. In particular we show that stable absolutely continuous spectrum covers the whole unit circle if $\Phi$ is trace class. We also observe that a trace formula in even powers of $\Phi$ holds for the relative index; it is quite useful in our application in chapter 4.

Theorem 2.1. Let $U$ be a unitary operator on a Hilbert space and $P$ an orthogonal projection. For the selfadjoint operator

$$
\Phi:=U^{*} P U-P=U^{*}[P, U]
$$

suppose that 1 is an isolated eigenvalue of finite multiplicity of $\Phi^{2}$ and define the integer

$$
\operatorname{ind}(\Phi):=\operatorname{dim} \operatorname{ker}(\Phi-\mathbb{I})-\operatorname{dim} \operatorname{ker}(\Phi+\mathbb{I}) .
$$

If the index does not vanish, ind $(\Phi)=n \neq 0$, then:

there exists a unitary $\widehat{U}$ such that $\widehat{U}=S \oplus \widetilde{U}$ where $S$ is a bilateral shift of multiplicity $|n|, \widetilde{U}$ is unitary on its subspace, it leaves $P$ invariant : $[\widetilde{U}, P]=0$ and

1. $\|U-\widehat{U}-F\|=\mathcal{O}\left(\left\|\Phi_{<}\right\|\right)$for a finite rank operator $F$ and $\Phi_{<}$the restriction of $\Phi$ to its spectral subspace off $\pm 1: \Phi_{<}:=\Phi \chi\left(\Phi^{2}<1\right)$;

2. if $[P, U]$ is compact then $U-\widehat{U}$ is compact and the essential spectrum of $U$ is the whole unit circle:

$$
\sigma(U)=S^{1}
$$

3. if $[P, U]$ is trace class then $U-\widehat{U}$ is trace class and the absolutely continuous spectrum of $U$ is the whole unit circle:

$$
\sigma_{a c}(U)=S^{1}
$$

Remark that the occurence of a wandering subspace for $U$ in this context as well as item (2) of Theorem 2.1 was observed by [10]. We proved item (3) for a special case in [4]. For the proof and for our applications we shall use the following facts and concepts, with the notation $P^{\perp}:=1-P$ : 
Proposition 2.2. $\quad 1$.

$$
\begin{aligned}
\Phi^{2} \leq 1 ; & {\left[\Phi^{2}, P\right]=0 ; } \\
\operatorname{ker}(\Phi+\mathbb{I})=\operatorname{ker}(P U P \uparrow \operatorname{Ran} P) ; & \operatorname{ker}(\Phi-\mathbb{I})=\operatorname{ker}\left(P^{\perp} U P^{\perp} \uparrow \operatorname{Ran} P^{\perp}\right) .
\end{aligned}
$$

2.

$$
\operatorname{ind}(\Phi)=\operatorname{trace}\left(\Phi^{2 j+1}\right)
$$

for any odd integer $2 j+1$ for which $\Phi^{2 j+1}$ is trace class.

3. If $\operatorname{ind}(\Phi)$ is defined then

$$
\operatorname{ind}(\Phi)=\operatorname{dim} \operatorname{ker}\left(\left(\Phi^{2}-\mathbb{I}\right) \uparrow \operatorname{Ran} P^{\perp}\right)-\operatorname{dim} \operatorname{ker}\left(\left(\Phi^{2}-\mathbb{I}\right) \uparrow \operatorname{Ran} P\right) .
$$

Furthermore:

$$
\operatorname{ind}(\Phi)=\operatorname{trace}\left(\left(P^{\perp}-P\right) \Phi^{2 j}\right)
$$

for any even integer $2 j$ for which $\Phi^{2 j}$ is trace class; in particular if $\Phi^{2}$ is trace class then

$$
\operatorname{ind}(\Phi)=\operatorname{trace}\left(P^{\perp} U^{*} P U P^{\perp}-P U^{*} P^{\perp} U P\right) .
$$

4. If $[0,1] \ni t \rightarrow U(t)$ is norm continuous and unitary and for $\Phi(t)=U^{*}(t) P U(t)$ : $1 \notin \sigma_{\text {ess }}\left(\Phi(t)^{2}\right)$ then $\mathbb{Z} \ni$ ind $(\Phi(t))=$ const. If $\Phi\left(t_{0}\right)^{j}$ is trace class for a $j \in \mathbb{N}$ then ind $(\phi(t))$ can be calculated by a trace as in item (2) or (3).

5. A d-dimensional subspace $\mathbb{L}$ is called wandering for a unitary $U$, if $U^{k} \mathbb{L} \perp$ $\mathbb{L} \quad \forall k \in \mathbb{N}$. For an orthogonal decomposition $\mathbb{L}=\bigoplus_{j=1}^{d} \mathbb{L}_{j}$ into 1-dimensional subspaces and for the $U$-invariant subspace

$$
\mathbb{M}:=\bigoplus_{k \in \mathbb{Z}} U^{k} \mathbb{L}=\bigoplus_{j=1}^{d} \bigoplus_{k \in \mathbb{Z}} U^{k} \mathbb{L}_{j}
$$

it holds that the restriction $U \uparrow \mathbb{M}$ is a bilateral shift of multiplicity $d$ and $U \uparrow \mathbb{M}^{\perp}$ is unitary on $\mathbb{M}^{\perp}$. In particular $\sigma_{a c}(U)=\mathbb{S}^{1}$.

\section{Remark 2.3.}

The assertions 1,2,5 are well known, see [7,23]. We contribute the supertrace formula for even powers of $\Phi$ for the index, i.e. item (3). This is in fact a generalization of Kitaev's formula [18], which says for the case of $P$ a multiplication operator in $\ell^{2}(\mathbb{Z} ; \mathbb{C})$ :

$$
\operatorname{ind}(\Phi)=\operatorname{trace}\left(\left(P^{\perp}-P\right) \Phi^{2}\right)=\sum_{x \in P, y \in P^{\perp}}\left|U_{x y}\right|^{2}-\left|U_{y x}\right|^{2} .
$$

We have formulated the result (3) for $\Phi=U^{*} P U-P$, but, as for the result (2), see [7], the proof works in the general case where $\Phi$ is the difference of two projections $\Phi=R-P$. 
Proof. of Proposition (2.2). Item(4) follows from items $(2,3)$ and the stability of the index, c.f. [7].

Item (3): $\psi \in \operatorname{ker}(\Phi+\mathbb{I}) \Longleftrightarrow\left(\psi \in \operatorname{ker}\left(\Phi^{2}-\mathbb{I}\right)\right.$ and $\left.\psi=P \psi\right) ; \psi \in \operatorname{ker}(\Phi-\mathbb{I}) \Longleftrightarrow$ $\left(\psi \in \operatorname{ker}\left(\Phi^{2}-\mathbb{I}\right)\right.$ and $\left.\psi=P^{\perp} \psi\right)$ which implies the first assertion.

Denote the projection $R:=U^{*} P U$ and $B:=R^{\perp}-P$. Then $\Phi=R-P$ and

$$
\Phi+B=P^{\perp}-P .
$$

Denote $Q_{\lambda^{2}}$ the spectral projection on $\operatorname{ker}\left(\Phi^{2}-\lambda^{2}\right)$ whose dimension is finite, $\Phi^{2}$ being compact. From [7] we know that $\Phi^{2}+B^{2}=\mathbb{I}$ and $\{\Phi, B\}=0$ and by their Theorem 4.2 that:

$$
\operatorname{trace}\left(Q_{\lambda^{2}}(\Phi+B)\right)=0 \text { if } 0<\lambda^{2}<1
$$

Assuming that $\Phi^{2 j}$ is trace class and calculating the trace in the spectral decomposition of $\Phi^{2}$ we have

$$
\begin{gathered}
\operatorname{trace}\left(\left(P^{\perp}-P\right) \Phi^{2 j}\right)=\sum_{\lambda^{2} \in \sigma\left(\Phi^{2}\right), \lambda^{2}<1} \lambda^{2 j} \underbrace{\operatorname{trace}\left(Q_{\lambda^{2}}(\Phi+B)\right)}_{0}+\operatorname{trace}\left(Q_{1}(\Phi+B)\right) \\
=\operatorname{trace}\left(Q_{1}(\Phi+B)\right)=\operatorname{trace}\left(Q_{1} \Phi\right)=\operatorname{dim} \operatorname{ker}(\Phi-\mathbb{I})-\operatorname{dim} \operatorname{ker}(\Phi+\mathbb{I}) .
\end{gathered}
$$

Proof. of Theorem 2.1 Following the idea of [10] we show that there exists a perturbation of $U$ which admits an $|n|$ dimensional wandering subspace $\mathbb{L}$. We freely make use of Proposition (2.2).

For the dimensions of the \pm 1 eigenspaces of $\Phi$ we can suppose $n=n_{+}-n_{-}<0$ (else consider $P^{\perp}$ in place of $P$ as $-\Phi=U^{*} P^{\perp} U-P^{\perp}$ ). Then

$$
\operatorname{dim} \operatorname{ker}(\Phi+\mathbb{I})=\operatorname{dim} \operatorname{ker}(\Phi-\mathbb{I})+|n|=n_{+}+|n| .
$$

Choose an $|n|$ dimensional subspace

$$
\mathbb{L} \subset \operatorname{ker}(\Phi+\mathbb{I})=\operatorname{ker}(P U P \uparrow \operatorname{Ran} P)
$$

and denote its $n_{+}$dimensional complement $\mathbb{L}^{\perp}:=\operatorname{ker}(\Phi+\mathbb{I}) \ominus \mathbb{L} \subset \operatorname{Ran} P$. The orthogonal projection $Q_{-}$on $\operatorname{ker}(\Phi+\mathbb{I})$ then decomposes as $Q_{-}=Q_{\mathbb{L}}+Q_{\mathbb{L}^{\perp}}$. $Q_{+}$ projects on $\operatorname{ker}(\Phi-\mathbb{I}) \subset \operatorname{Ran} P^{\perp}$ and $Q_{0}:=\mathbb{I}-Q_{+}-Q_{-}$. Note that $Q_{0}=$ $\chi\left(\Phi^{2} \in[0,1)\right)$, the spectral projection off 1 .

Choose a $n_{+}$dimensional unitary $V$

$$
V: \mathbb{L}^{\perp} \rightarrow \operatorname{ker}(\Phi-\mathbb{I})=\operatorname{Ran} Q_{+}=\operatorname{ker}\left(P^{\perp} U P^{\perp} \uparrow \operatorname{Ran} P^{\perp}\right)
$$


Observe for the diagonal operator :

$$
P U P+P^{\perp} U P^{\perp}=U-[P,[P, U]]=U(\underbrace{\mathbb{I}-\Phi^{2}-[P, \Phi]}_{=: W}) .
$$

Denoting the projection $R:=U^{*} P U$, it holds : $\Phi=R-P, \mathbb{I}-\Phi^{2}=P^{\perp} R^{\perp}+R P$, $W=R^{\perp} P^{\perp}+R P$. It follows

$$
W^{*} W=P^{\perp} R^{\perp} P^{\perp}+P R P=\mathbb{I}-\Phi^{2} .
$$

$W$ commutes with $\Phi^{2}$ thus with $Q_{0}$ because $P$ does. Denote $U_{0}: \operatorname{Ran} Q_{0} \rightarrow \operatorname{Ran} Q_{0}$ the unitary such that

$$
W=U_{0}\left(\mathbb{I}-\Phi^{2}\right)^{1 / 2} \text { on } \operatorname{Ran} Q_{0}
$$

and $\widehat{U}$ the unitary on the entire Hilbert space :

$$
\widehat{U}\left(\psi_{\mathbb{L}}+\psi_{-}+\psi_{+}+\psi_{0}\right):=U\left(\psi_{\mathbb{L}}+V^{*} \psi_{+}+V \psi_{-}+U_{0} \psi_{0}\right)
$$

for $\psi_{\mathbb{L}}+\psi_{-}+\psi_{+}+\psi_{0} \in \operatorname{Ran} Q_{\mathbb{L}}+\operatorname{Ran} Q_{\mathbb{L}}^{\perp}+\operatorname{Ran} Q_{+}+\operatorname{Ran} Q_{0}$.

By construction we have (see also figure 1 ):

$$
\begin{aligned}
& \psi_{\mathbb{L}} \in \operatorname{ker} P U P \Rightarrow \psi_{\mathbb{L}} \in \operatorname{ker} P \widehat{U} P \\
& \psi_{-} \in \operatorname{ker} P U P \Rightarrow V \psi_{-} \in \operatorname{ker} P^{\perp} U P^{\perp} \Rightarrow \widehat{U} \psi_{-}=\widehat{U} P \psi_{-}=P \widehat{U} P \psi_{-}, \\
& \psi_{+} \in \operatorname{ker} P^{\perp} U P^{\perp} \Rightarrow V^{*} \psi_{+} \in \operatorname{ker} P U P \Rightarrow \widehat{U} \psi_{+}=\widehat{U} P^{\perp} \psi_{+}=P^{\perp} \widehat{U} P^{\perp} \psi_{+}, \\
& \widehat{U} Q_{0}=\left(P U P+P^{\perp} U P^{\perp}\right)\left(\mathbb{I}-\Phi^{2}\right)^{-1 / 2} Q_{0}=\left(P \widehat{U} P+P^{\perp} \widehat{U} P^{\perp}\right) Q_{0} \\
& \quad \text { as }\left[P, \Phi^{2}\right]=0 .
\end{aligned}
$$

It follows

$$
\operatorname{ker}(P \widehat{U} P \uparrow \operatorname{Ran} P)=\mathbb{L} ; \quad \operatorname{ker}\left(P^{\perp} \widehat{U} P^{\perp} \uparrow \operatorname{Ran} P^{\perp}\right)=0 ; \quad P \widehat{U} P^{\perp}=0 .
$$

This implies for all $k \in \mathbb{N}$

$$
P \widehat{U}^{k} P^{\perp}=0, \quad P \widehat{U}^{k} Q_{\mathbb{L}}=0, \quad \text { and in particular } \quad Q_{\mathbb{L}} \widehat{U}^{k} Q_{\mathbb{L}}=0,
$$

thus $\mathbb{L}$ is a wandering subspace for $\widehat{U}$. This implies for the invariant subspace $\mathbb{M}:=$ $\bigoplus_{\mathbb{Z}} \widehat{U}^{k} \mathbb{L}$ that the restriction $S:=\widehat{U} \uparrow \mathbb{M}$ is a bilateral shift of multiplicity $|n|$ and that $\widetilde{U}:=\widehat{U} \uparrow \mathbb{M}^{\perp}$ is unitary.

Remark that $\widehat{U}\left(\mathbb{I}-Q_{\mathbb{L}}\right)$ commutes with $P$ and in particular $\left[Q_{\mathbb{M}}, P\right]=0$ which implies $[\widetilde{U}, P]=0$.

Concerning the regularity remark that

$$
U^{*} \widehat{U}-\mathbb{I}=\underbrace{\left(V^{*}-\mathbb{I}\right) Q_{+}+(V-\mathbb{I}) Q_{\mathbb{L}^{\perp}}}_{=: F}+\left(U_{0}-\mathbb{I}\right) Q_{0}
$$




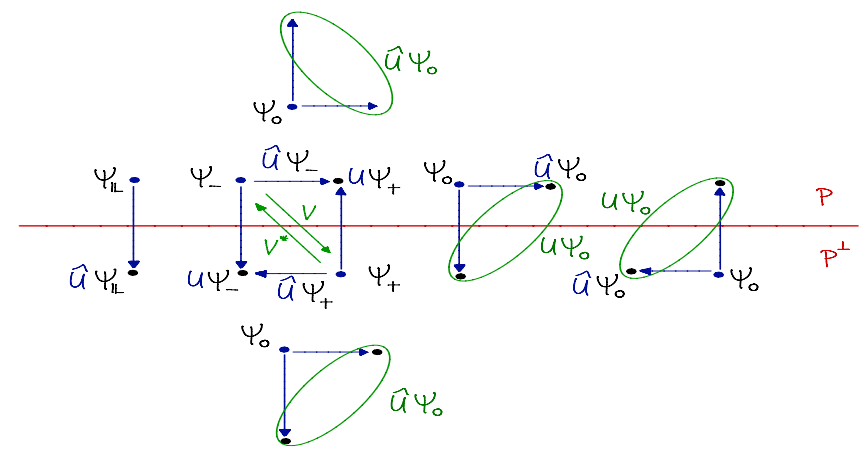

Figure 1: The action of $\widehat{U}$

where $F$ is of finite rank, and, as operators on $\operatorname{Ran} Q_{0}$ :

$$
\left(U_{0}-\mathbb{I}\right)\left(\mathbb{I}-\Phi^{2}\right)^{1 / 2}=W-\left(\mathbb{I}-\Phi^{2}\right)^{1 / 2}=-\Phi^{2}-[P, \Phi]+\Phi^{2}\left(\mathbb{I}+\left(\mathbb{I}-\Phi^{2}\right)^{1 / 2}\right)^{-1}
$$

which is of order $\mathcal{O}\left(\left\|\Phi \chi\left(\Phi^{2}<1\right)\right\|\right)$ and compact (trace class) if $\Phi$ is compact (trace class).

The results on the spectrum of $U$ then follow from invariance of the essential (resp. ac) spectrum under compact (resp. trace class) perturbations.

\section{Engineering Quantum Walks with prescribed cur- rents on bulk boundaries}

We consider coined quantum walks on $\mathbb{H}=\ell^{2}\left(\mathbb{Z}^{d}, \mathbb{C}^{2 d}\right)$, with scalar product $\langle\cdot, \cdot\rangle$. Denote the discrete unit sphere by

$$
I_{2 d}:=S^{d-1} \cap \mathbb{Z}^{d}
$$

and its directions by $( \pm j):= \pm e_{j}, j \in\{1, \ldots, d\}$, with the canonical basis vectors $e_{j} \in \mathbb{Z}^{d}$; thus $I_{2 d}=\{( \pm j)\}$. A quantum direction is a basis vector $| \pm j\rangle \in \mathbb{C}^{2 d}$ with $|+j\rangle:=\widehat{e}_{2 j-1},|-j\rangle:=\widehat{e}_{2 j}$ for the canonical basis vectors $\widehat{e}_{j} \in \mathbb{C}^{2 d}$; we freely use the bijection from classical to quantum directions : $( \pm j) \leftrightarrow| \pm j\rangle$.

For $\tau \in I_{2 d}$ denote

$$
P_{\tau}:=|\tau\rangle\langle\tau|
$$

the orthogonal projection on $\operatorname{span}(|\tau\rangle) \subset \mathbb{C}^{2 d}$.

By a multiplication operator on $\mathbb{H}$ with symbol $M$ we understand

$$
\mathbf{M} \psi(x)=M(x) \psi(x), \quad \psi \in \mathbb{H}, \quad M(x) \in \mathbb{B}\left(\mathbb{C}^{2 d}\right) .
$$


Denote $S_{\tau}$ the right shift $S_{\tau} \psi(x):=\psi(x-\tau)$. The conditional shift operator is

$$
\mathbf{T}: \mathbb{H} \rightarrow \mathbb{H}, \quad \mathbf{T}:=\sum_{\tau \in I_{2 d}} S_{\tau} \mathbf{P}_{\tau}
$$

where $\mathbf{P}_{\tau}$ has the symbol $P_{\tau}, \forall x$, i.e.: $\mathbf{T} \psi(x)=\sum_{\tau}\langle\tau, \psi(x-\tau)\rangle|\tau\rangle$.

By a simple quantum walk $U: \mathbb{H} \rightarrow \mathbb{H}$ with coin $\mathbf{C}$ we understand

$$
U=\mathbf{T C} \quad \text { with } \mathbf{C} \text { a multiplication operator with unitary symbol . }
$$

Thus the corresponding one step unitary evolution $U$ of the walker is so that the coin matrices first reshuffle or update the coin states so that the pieces of the wave function corresponding to different internal states are then shifted to different directions, depending on the internal state : $U \psi(x)=\sum_{\tau}\langle\tau, C(x-\tau) \psi(x-\tau)\rangle|\tau\rangle$.

We are working with a flux operator defined for an adapted projection, i.e.: a projection which at each site projects onto quantum directions:

Definition 3.1. A projection valued multiplication operator $\mathbf{P}$ with symbol $P$ is called adapted if

$$
\left[P(x), P_{\tau}\right]=0, \quad x \in \mathbb{Z}^{d}, \tau \in I_{2 d}
$$

The set of open directions at $x \in \mathbb{Z}^{d}$ is defined as

$$
I_{\mathbf{P}}(x):=\left\{\tau \in I_{2 d},\langle\tau, P(x) \tau\rangle \neq 0\right\}
$$

so $P(x)=\sum_{\tau \in I_{\mathbf{P}}(x)}|\tau\rangle\langle\tau|$.

If $\mathbf{P}$ is an adapted projection then $\mathbf{T}^{*} \mathbf{P T}$ is also adapted with symbol

$$
\widehat{P}(x)=\sum_{\tau \in I_{2 d}} P_{\tau} P(x+\tau) P_{\tau} ;
$$

An adapted $\mathbf{P}$ is called homogeneous in $G \subset \mathbb{Z}^{d}$ if

$$
\operatorname{dim} \operatorname{Ran} \widehat{P}(x)=\operatorname{dim} \operatorname{Ran} P(x), \forall x \in G .
$$

Example 3.2. For $d=1, G=[1, \infty) \cap \mathbb{Z}$ the only two adapted projections homogeneous in $G$ and of $\operatorname{dim} \operatorname{Ran} P(x)=1, x \in G$ are given by

1. $P_{a}(x):=\chi(x \in G)|1\rangle\langle 1|$

2. $P_{b}(x):=\chi(x \in G)\left|(-1)^{x}\right\rangle\left\langle(-1)^{x}\right|$

then for $x \in G: \widehat{P}_{a}(x)=P_{a}(x), \widehat{P}_{b}(x)=P_{b}^{\perp}(x)$

Proposition 3.3. Let $U$ be a quantum walk with coin $\mathbf{C}, \mathbf{P}$ an adapted projection, $\Phi=U^{*} \mathbf{P} U-\mathbf{P}$. It holds 
1. the eigenvalue 1 of $\Phi^{2}$ is isolated and finitely degenerate iff there exists $R>0$ such that $\mathbf{P}$ is homogeneous in $\left\{x \in \mathbb{Z}^{d},|x|>R\right\}$ and it holds in $\mathbb{C}^{2 d}$ :

$$
\sup _{|x|>R}\left\|C^{*}(x) \widehat{P}(x) C(x)-P(x)\right\| \leq c<1 .
$$

In this case

$$
\operatorname{ind}(\Phi)=\sum_{|x| \leq R}(\operatorname{dim} \operatorname{Ran} \widehat{P}(x)-\operatorname{dim} \operatorname{Ran} P(x))
$$

2. $[U, \mathbf{P}]$ is compact iff $\lim _{|x| \rightarrow \infty}\left\|C^{*}(x) \widehat{P}(x) C(x)-P(x)\right\|=0$;

3. $\sum_{x \in \mathbb{Z}^{d}}\left\|C^{*}(x) \widehat{P}(x) C(x)-P(x)\right\|<\infty$ implies that $[U, \mathbf{P}]$ is trace class.

Proof. 1. $\Phi$ is an operator valued multiplication operator with self-adjoint symbol

$$
\Phi(x)=C^{*}(x) \widehat{P}(x) C(x)-P(x),
$$

for the spectrum it holds $\sigma(\Phi)=\bigcup_{x \in \mathbb{Z}^{2}} \sigma(\Phi(x))$. Also the eigenspace $\operatorname{ker}(\Phi \pm \mathbb{I})$ is the direct sum of $\operatorname{ker}(\Phi(x) \pm \mathbb{I})$ which is trivial for $x$ with $|x|>R$ as $\|\Phi(x)\|<1$ there. Thus $\operatorname{dim} \operatorname{ker}(\Phi \pm \mathbb{I})=\sum_{|x| \leq R} \operatorname{dim} \operatorname{ker}(\Phi(x) \pm \mathbb{I})$ which implies the formula for the index.

2. Compactness of the multiplication operator $\Phi$ is equivalent to $\|\Phi(x)\| \rightarrow 0$.

3. Follows from lemma 3.4

Lemma 3.4. Let $\mathrm{M}$ be a multiplication operator. If its symbol satisfies

$$
\sum_{x \in \mathbb{Z}^{d}}\|M(x)\|<\infty
$$

then $\mathbf{M}$ is trace class.

Proof. $\mathbf{M}$ is trace class iff $\sum_{x} \sum_{\mu} \lambda_{\mu}(x)=\sum_{x}\|M(x)\|_{t r}<\infty$ for the singular values of $M(x)$. The result follows from the equivalence of norms.

As a corollary from Theorem 2.1 and Proposition 3.3 we have

Corollary 3.5. Let $\mathbf{P}$ be a projection homogeneous in $\left\{x \in \mathbb{Z}^{d},|x|>R\right\}$ such that

$$
\sum_{x \in \mathbb{Z}^{d}} \operatorname{dim} \operatorname{Ran} \widehat{P}(x)-\operatorname{dim} \operatorname{Ran} P(x) \neq 0
$$

let $\mathbf{C}$ be a coin and $U=\mathbf{T C}$ the associated quantum walk. Then 
1. $C^{*}(x) \widehat{P}(x) C(x)-P(x) \rightarrow|x| \rightarrow \infty 0$ implies : $\sigma(U)=S^{1}$;

2. $\sum_{x}\left\|C^{*}(x) \widehat{P}(x) C(x)-P(x)\right\|<\infty$ implies : $\sigma_{a c}(U)=S^{1}$;

3. for $C(x)$ as in 2. and a second coin $\mathbf{C}_{2}$ such that $\sum_{x}\left\|C(x)-C_{2}(x)\right\|<\infty$ it holds

$$
\sigma_{a c}\left(\mathbf{T C}_{2}\right)=S^{1}
$$

Proof. 1. and 2. follow from Theorem 2.1, proposition 3.3 and Lemma 3.4. For the third assertion remark that $\mathbf{T C}_{2}-\mathbf{T} \mathbf{C}_{1}=\mathbf{T}\left(\mathbf{C}_{2}-\mathbf{C}_{1}\right)$ which is trace class, and the claim follows from the Birman-Krein theorem.

Our basic example, see figure (2) and example (3.2), in one dimension is

Example 3.6. (Basic example) For dimension $d=1$ let $\mathbf{C}$ be a coin such that $\left[C(x), P_{(1)}\right]=0 \forall x \geq N \geq 0$ then $\sigma_{a c}(\mathbf{T C})=S^{1}$.

Proof. Let $\mathbf{P}$ be an adapted projection such that $P(x)=0$ for $x$ less or equal to $-N$ and $P(x)=P_{(1)}$ for $x$ greater or equal to $N$.Then for all directions $\tau: P(x+\tau)=$ $P(x), \forall|x|>N$ and $C^{*}(x) \widehat{P}(x) C(x)-P(x)=\Phi(x)=0, \forall|x|>N$. So $\Phi$ is trace class and

$$
\begin{gathered}
\operatorname{trace}(\Phi)=\sum_{|x| \leq N} \sum_{\tau}\left(\operatorname{trace}\left(P_{\tau} P(x+\tau) P_{\tau}\right)-\operatorname{trace}\left(P_{\tau} P(x) P_{\tau}\right)\right. \\
=\operatorname{trace}\left(P_{(1)}(P(N+1)-P(-N))\right)+\operatorname{trace}\left(P_{(-1)}(P(-N-1)-P(N))\right)=\operatorname{trace}\left(P_{(1)}\right)=1
\end{gathered}
$$

and the claim follows from Theorem (2.1).

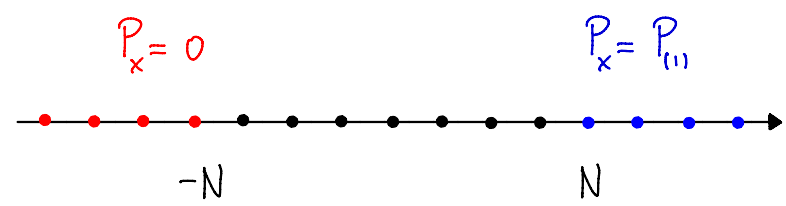

Figure 2: Basic example

Remark that the above quantum walk TC $\uparrow \chi([N, \infty)) P_{1}$ is a unilateral shift and the coin is totally arbitrary on the left of $N$. In particular other spectral type may coexist with the absolutely continuous spectum on $S^{1}$. 


\subsection{Networks of leads in $\mathbb{Z}^{d}$}

The basic example above is effortlessly transported to a halfline in $\mathbb{Z}^{d}$.

Example 3.7. (Halfline in $\mathbb{Z}^{d}$ ) Denote $\mathbb{N}_{(1)}:=\mathbb{N} \times\{0\} \times \cdots \times\{0\} \subset \mathbb{Z}^{d}$. Let $\mathbf{C}$ be a coin and $U=\mathbf{T C}$ the associated quantum walk.

1. If $\left[C(x), P_{(1)}\right]=0, \forall x \in \mathbb{N}_{(1)}$ then it holds with the symbol $P(x):=\chi(x \in$ $\left.\mathbb{N}_{(1)}\right) P_{(1)}: \operatorname{ind}\left(U^{*} \mathbf{P} U-\mathbf{P}\right)=1$.

2. If $\left[C(x), P_{(-1)}\right]=0, \forall x \in \mathbb{N}_{(1)}$ then it holds with the symbol $P(x):=\chi(x \in$ $\left.\mathbb{N}_{(1)}\right) P_{(-1)}:$ ind $\left(U^{*} \mathbf{P} U-\mathbf{P}\right)=-1$.

Proof. It is sufficient to calculate $\widehat{P}$ in a 1-neighborhood of $\mathbb{N}_{(1)}$ because $\Phi(x)$ vanishes outside. Here we have, see figure (3)

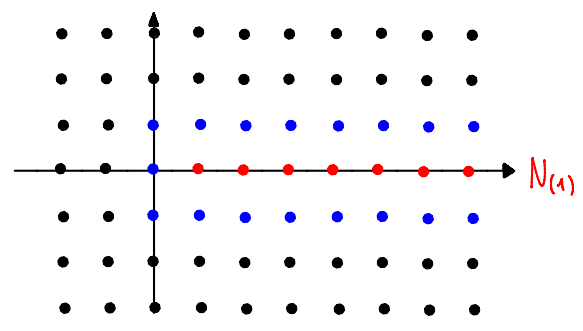

Figure 3: 1-neighborhood

1.

$$
\begin{gathered}
P(x+(1))=\left\{\begin{array}{cl}
P_{(1)} & x \in \mathbb{N}_{(1)} \cup\{(0,0, \ldots)\} \\
0 & \text { else }
\end{array}\right. \\
P(x+(-1))=\left\{\begin{array}{cc}
P_{(1)} & x \in \mathbb{N}_{(1)} \backslash\{(1,0, \ldots)\} \\
0 & \text { else }
\end{array}\right. \\
P(x+(2))=\left\{\begin{array}{cc}
P_{(1)} & x \in \mathbb{N}_{(1)}+(-2) \\
0 & \text { else }
\end{array}\right. \\
P(x+(-2))=\left\{\begin{array}{cc}
P_{(1)} & x \in \mathbb{N}_{(1)}+(2) \\
0 & \text { else }
\end{array}\right.
\end{gathered}
$$

it follows $\widehat{P}(x)=P_{(1)} \chi\left(x \in \mathbb{N}_{(1)} \cup\{(0,0, \ldots)\}\right)$ so $\mathbf{P}$ is homogeneous in $\mathbb{Z}^{d} \backslash$ $\{(0,0, \ldots)\}$ and by formula $(4): \operatorname{ind}(\Phi)=\operatorname{rank}\left(P_{1} \chi(x=(0,0, \ldots))=1\right.$.

2. In the second case

$\widehat{P}(x)$ equals $P(x)$ on $\mathbb{N}_{(1)} \backslash\{(1,0, \ldots)\}, \mathbf{P}$ is homogeneous in $\mathbb{Z}^{d} \backslash\{(1,0, \ldots)\}$ and

$\operatorname{ind}(\Phi)=-\operatorname{rank}\left(P_{(-1)} \chi(x=(1,0, \ldots))\right)=-1$. 
Generalising this example we consider outgoing and incoming leads :

Definition 3.8. A path $\gamma: \mathbb{Z} \supset G \rightarrow \mathbb{Z}^{d}$ is called regular if $|\overrightarrow{\gamma(t-1) \gamma(t)}|=1, \forall t-$ $1, t \in G$ and simple if it is injective. An unbounded regular path is called a (classical) lead. $A$ lead is outgoing if $G=\mathbb{N}=\{1,2, \ldots\}$ and a lead is incoming if $G=-\mathbb{N}=$ $\{\ldots,-2,-1\}$.

The tangent path of $\gamma$ is

$$
\tau_{\gamma}: G \rightarrow I_{2 d}, \quad \tau_{\gamma}(t):=\overrightarrow{\gamma(t-1) \gamma(t)}
$$

with the convention : $\tau_{\gamma}(1):=\tau_{\gamma}(2)$ for an outgoing lead. An outgoing or incoming lead is called admissible if it has no tangential selfintersections, i.e.

$$
G \ni t \rightarrow\left(\gamma(t), \tau_{\gamma}(t)\right) \in \mathbb{Z}^{d} \times I_{2 d}
$$

is injective.

We use the following notations : for $x_{0} \in \mathbb{Z}^{d}, \tau \in I_{2 d}:\left|x_{0}, \tau_{0}\right\rangle \in \ell^{2}\left(\mathbb{Z}^{d}, \mathbb{C}^{2 d}\right)$ is defined by $x \mapsto \delta_{x, x_{0}}\left|\tau_{0}\right\rangle$ and

$$
\left|x_{0}, \tau_{0}\right\rangle\left\langle x_{0}, \tau_{0}\right|
$$

for the orthogonal projection on $\operatorname{span}\left\{\left|x_{0}, \tau_{0}\right\rangle\right\}$.

Definition 3.9. Let $\gamma: G \rightarrow \mathbb{Z}^{d}$ be a classical lead and $\tau_{\gamma}$ its tangent. The associated quantum lead is an adapted projection with symbol along $\gamma$, i.e.

$$
P_{\gamma}(t)=\left|\gamma(t), \tau_{\gamma}(t)\right\rangle\left\langle\gamma(t), \tau_{\gamma}(t)\right|, \quad t \in G .
$$

We construct quantum walks which implement the time evolution along a lead:

Proposition 3.10. Let $P_{\gamma}$ be an admissible outgoing (resp. incoming) quantum lead and let $\mathbf{C}$ be a coin such that

$$
\left|\left\langle\tau_{\gamma}(t+1), C(\gamma(t)) \tau_{\gamma}(t)\right\rangle\right|=1 \quad \forall t \in G \text { s.t. } t+1 \in G .
$$

Then $P_{\gamma}(t) P_{\gamma}(s)=0, \forall t \neq s \in G$ and for the quantum walk $U=\mathbf{T C}$ it holds:

$$
\begin{aligned}
U^{n} P_{\gamma}(1) U^{* n} & =P_{\gamma}(1+n) \quad \forall n \in \mathbb{N} \text { if } \gamma \text { is outgoing }, \\
U^{* n} P_{\gamma}(-1) U^{n} & =P_{\gamma}(-1-n) \quad \forall n \in \mathbb{N} \text { if } \gamma \text { is incoming. }
\end{aligned}
$$

In particular $\operatorname{Ran} P_{\gamma}(1)$ (resp. $\left.\operatorname{Ran} P_{\gamma}(-1)\right)$ is a wandering subspace for $U$ (resp. $U^{*}$ ) if $\gamma$ is outgoing (resp. incoming), and for the total projector

$$
\mathbf{P}_{\gamma}:=\sum_{t \in G} P_{\gamma}(t) \text { and the flux } \Phi=U^{*} \mathbf{P}_{\gamma} U-\mathbf{P}_{\gamma}, \quad G=\mathbb{N}(\text { resp. } G=-\mathbb{N})
$$

it holds

$$
\operatorname{ind}(\Phi)=\left\{\begin{aligned}
1 & \text { if } \gamma \text { is outgoing } \\
-1 & \text { if } \gamma \text { is incoming }
\end{aligned}\right.
$$


Proof. By the admissibility assumptions $\left|\gamma\left(t_{0}\right), \tau_{\gamma}\left(t_{0}\right)\right\rangle \perp\left|\gamma\left(t_{1}\right), \tau_{\gamma}\left(t_{1}\right)\right\rangle, \forall t_{0}, t_{1} \in G$. It follows that $\mathbf{P}_{\gamma}$ is well defined as a strong limit of orthogonal projections.

For any $t \in G$ ( except $t=-1$ in the incoming case) it holds for a phase $\varphi_{t} \in S^{1}$ :

$$
U\left|\gamma(t), \tau_{\gamma}(t)\right\rangle=\varphi_{t} \mathbf{T}\left|\gamma(t), \tau_{\gamma}(t+1)\right\rangle=\varphi_{t}\left|\gamma(t+1), \tau_{\gamma}(t+1)\right\rangle .
$$

This proves the wandering subspace property and furthermore:

$$
U \mathbf{P}_{\gamma} U^{*}= \begin{cases}\mathbf{P}_{\gamma}-\left|\gamma(1), \tau_{\gamma}(1)\right\rangle\left\langle\gamma(1), \tau_{\gamma}(1)\right| & \text { if } \gamma \text { is outgoing } \\ \mathbf{P}_{\gamma}+U\left|\gamma(-1), \tau_{\gamma}(-1)\right\rangle\left\langle\gamma(-1), \tau_{\gamma}(-1)\right| U^{*} & \text { if } \gamma \text { is incoming. }\end{cases}
$$

It follows for $\Phi=U^{*} \mathbf{P}_{\gamma} U-\mathbf{P}_{\gamma}$ :

$$
\Phi= \begin{cases}U^{*}\left|\gamma(1), \tau_{\gamma}(1)\right\rangle\left\langle\gamma(1), \tau_{\gamma}(1)\right| U & \text { if } \gamma \text { is outgoing } \\ -\left|\gamma(-1), \tau_{\gamma}(-1)\right\rangle\left\langle\gamma(-1), \tau_{\gamma}(-1)\right| & \text { if } \gamma \text { is incoming }\end{cases}
$$

and

$$
\operatorname{ind}(\Phi)=\left\{\begin{aligned}
1 & \text { if } \gamma \text { is outgoing } \\
-1 & \text { if } \gamma \text { is incoming }
\end{aligned}\right.
$$

Because of the locality or the construction we can consider an arbitrary finite number of leads and design currents.

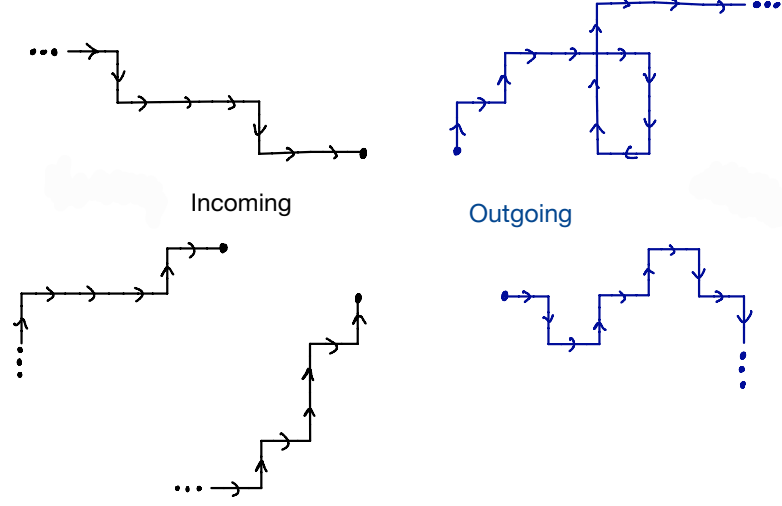

Figure 4: Networks of leads

Definition 3.11. We say that leads do not cross tangentially if for any $(x, \tau) \in \mathbb{Z}^{d} \times I_{2 d}$ there is at most one lead $\gamma$ such that $(x, \tau) \in \operatorname{Ran}\left(\gamma, \tau_{\gamma}\right)$

Proposition 3.12. For $n_{i}, n_{o} \in \mathbb{N}$ consider $n_{i}$ incoming leads $\gamma_{j}$ and $n_{o}$ outgoing leads $\rho_{k}$ which do not cross tangentially. Let $\mathbf{C}$ be a coin satisfying condition (5) along all 
leads and $U=\mathbf{T C}$ the associated walk. Then the total projections on the quantum leads are mutually orthogonal and for

$$
\mathbf{P}=\sum_{j} P_{\gamma_{j}}+\sum_{k} P_{\rho_{k}} \text { and the flux } \Phi=U^{*} \mathbf{P} U-\mathbf{P}
$$

it holds

$$
\operatorname{ind}(\Phi)=n_{o}-n_{i} .
$$

Proof. At a given $x \in \mathbb{Z}^{d}$ no more than $2 d$ paths can cross non tangentially so there exists a $C(x) \in U(2 d)$ satisfying (5). The non-tangential condition implies $P_{\alpha}(t) P_{\beta}(s)=0, \forall t \neq s, \forall \alpha=\beta \in\left\{\gamma_{j}, \rho_{k}\right\}$. The result then follows because equation (6) implies: $\operatorname{ind}\left(\Phi_{P_{1}+P_{2}}\right)=\operatorname{ind}\left(\Phi_{1}\right)+\operatorname{ind}\left(\Phi_{2}\right)$

Remarks 3.13. 1. Note that the coin $C(x)$ is arbitrary for $x$ outside the leads and that $n_{o}-n_{i} \neq 0$ implies $\sigma_{a c}(U)=S^{1}$; also the spectral result remains true if condition (5) is only satisfied asymptotically in the sense of Corollary 3.5, point 3.

2. Note that $\sigma_{a c}=S^{1}$ as soon as a quantum lead is present such that condition (5) is satisfied.

Examples 3.14. In $\mathbb{Z}^{2}$ consider

1. two outgoing leads: $\rho_{1}(n)=(0, n-1), \forall n \in \mathbb{N}, \rho_{2}(n)=(-n+1,0), \forall n \in \mathbb{N}$ with tangents $\tau_{\rho_{1}}(n)=(0,1), \tau_{\rho_{2}}(n)=(-1,0)$. Then for $\Phi=U^{*}\left(P_{\rho_{1}}+\right.$ $\left.P_{\rho_{2}}\right) U-\left(P_{\rho_{1}}+P_{\rho_{2}}\right):$ ind $(\Phi)=2$;

2. an incoming and an outgoing lead: $\gamma_{1}(n)=(0,-n-1), \forall n \in-\mathbb{N}, \rho_{2}(n)=$ $(-n+1,0), \forall n \in \mathbb{N}$ with tangents $\tau_{\gamma_{1}}(n)=(0,-1), \tau_{\rho_{2}}(n)=(-1,0)$. Then for $\Phi=U^{*}\left(P_{\gamma_{1}}+P_{\rho_{2}}\right) U-\left(P_{\gamma_{1}}+P_{\rho_{2}}\right)$ : ind $(\Phi)=1-1=0$.

\subsection{Networks of leads tangential to bulk boundaries}

We now design quantum walks which propagate along a network of leads on a surface bounding a half-space and such that there is no flow out of the halfspace. We start by constructing a projection and a coin such that there is no flow out the halfspace and add conducting leads on the surface in a second step.

Consider the lattice

$\mathbb{Z}^{d+1}$ split in two halfs $\mathbb{Z}_{ \pm}^{d+1}:=\mathbb{Z}^{d} \times( \pm \mathbb{N})$ separated by the boundary $\Gamma=\mathbb{Z}^{d} \times\{0\}$.

The tangent space is

$T \Gamma=\{( \pm j), j \in\{1, \ldots, d\}\} \subset I_{2(d+1)}$, and the in-(out-)ward normal $\pm N=( \pm(d+1))$; 


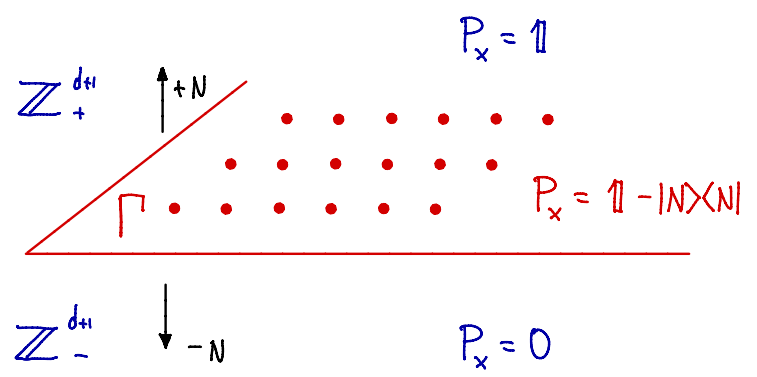

Figure 5: Splitting by a hypersurface

we use the same notations for the quantum directions $T \Gamma=\{| \pm j\rangle\}_{j \in\{1, \ldots, d\}} \subset$ $\mathbb{C}^{2(d+1)},| \pm N\rangle=| \pm(d+1)\rangle$

Lemma 3.15. Let $\mathbf{P}$ be the adapted projection with symbol

$$
P(x)=\left\{\begin{array}{lr}
\mathbb{I} & x \in \mathbb{Z}_{+}^{d+1} \\
0 & x \in \mathbb{Z}_{-}^{d+1} \\
|-N\rangle\left\langle-N\left|+P_{T \Gamma}=\mathbb{I}-\right| N\right\rangle\langle N| & x \in \Gamma
\end{array}\right.
$$

(with $P_{T \Gamma}$ the projection on the tangential directions) and $\mathbf{C}$ any coin whose symbol leaves the tangent space invariant and which reflects the normal on $\Gamma$, i.e.:

$$
C(x) T \Gamma=T \Gamma, C(x)| \pm N\rangle=|\mp N\rangle \quad \forall x \in \Gamma
$$

then $\mathbf{P}$ is homogeneous in $\mathbb{Z}^{d+1}$ and for $U=\mathbf{T C}$ it holds

$$
\Phi=U^{*} \mathbf{P} U-\mathbf{P}=0 .
$$

Note that because the coin is reflecting on the surface a Quantum Walker can move tangentially to, but cannot cross $\Gamma$.

Proof. By homogeneity it is sufficient to consider the 1-neighborhood of $\Gamma=\Gamma \cup(\Gamma+$ $N) \cup(\Gamma-N)$. For $x \in \Gamma$ we have

$$
\widehat{P}(x)=\sum_{\tau \in \Gamma} P_{\tau} \mathbb{I} P_{\tau}+P_{N} \underbrace{P(x+N)}_{=\mathbb{I}} P_{N}+P_{-N} \underbrace{P(x-N)}_{0} P_{-N}=\mathbb{I} \uparrow T \Gamma+P_{N},
$$

for $x \in \Gamma+N$ :

$$
\widehat{P}(x)=\sum_{\tau \in I_{2(d+1)} \backslash\{-N\}} P_{\tau}+\underbrace{P_{-N} P(x-N) P_{-N}}_{P_{-N}}=P(x)
$$


and for $x \in \Gamma-N$ :

$$
\widehat{P}(x)=P_{N} P(x+N) P_{N}=0=P(x) .
$$

It follows that $\operatorname{rank} \widehat{P}(x)=\operatorname{rank} P(x), \forall x$ and from the properties of $C$ :

$$
\Phi(x)=C^{*}(x) \widehat{P}(x) C(x)-P(x)=0, \forall x .
$$

We now are able to consider a network of leads on the surface $\Gamma$ and a quantum walk such that there is no crossing from $\mathbb{Z}_{+}^{d+1}$ to $\mathbb{Z}_{-}^{d+1}$ but which propagates on $\Gamma$ with arbitrary index of $\Phi$ :

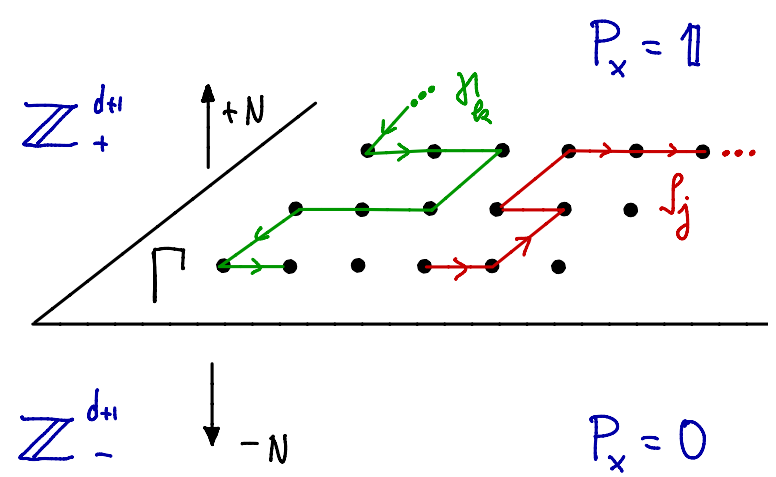

Figure 6: Leads on a surface

Theorem 3.16. For $n_{i}, n_{o} \in \mathbb{N}$ consider $n_{i}$-incoming leads $\gamma_{j}$ and $n_{o}$ outgoing leads $\rho_{k}$ on $\Gamma$ which are admissible and do not cross tangentially.

Let $\mathbf{P}$ be the adapted projection with symbol

$$
P(x)=\left\{\begin{array}{lr}
\mathbb{I} & x \in \mathbb{Z}_{+}^{d+1} \\
0 & x \in \mathbb{Z}_{-}^{d+1} \\
|-N\rangle\langle-N| & x \in \Gamma
\end{array}\right.
$$

and, as in proposition 3.12

$$
\mathbf{P}_{L}=\sum_{j} P_{\gamma_{j}}+\sum_{k} P_{\rho_{k}}
$$

the total projection on the leads. Let $\mathbf{C}$ be any coin which is reflecting on $\Gamma$ :

$$
C(x)| \pm N\rangle=|\mp N\rangle \quad \forall x \in \Gamma
$$


and such that condition (5) is verified for all leads. Then for

$$
Q=\mathbf{P}+\mathbf{P}_{L}, \quad \Phi=U^{*} Q U-Q
$$

it holds

$$
\operatorname{ind}(\Phi)=n_{0}-n_{i}
$$

Furthermore

1. $U \ell^{2}\left(\mathbb{Z}_{ \pm}^{d+1} ; \mathbb{C}^{2 d}\right) \subset \ell^{2}\left(\mathbb{Z}_{ \pm}^{d+1} \times \Gamma ; \mathbb{C}^{2 d}\right)$

2. $\operatorname{Ran} P_{\rho_{k}}(1)$ is a wandering subspace for $U$

3. $\operatorname{Ran} P_{\gamma_{j}}(-1)$ is a wandering subspace for $U^{*}$

Proof. $\mathbf{P}_{L}$ contains only tangential directions while $P$ contains none, $\operatorname{ind}\left(\Phi_{\mathbf{P}_{L}}\right)+$ $\operatorname{ind}\left(\Phi_{\mathbf{P}}\right)=\operatorname{ind}\left(\Phi_{Q}\right)$. For the same reasons the two conditions on the coin are compatible and the dynamical results follow from the previous results.

Remark 3.17. 1. Note that the coin $C(x)$ is arbitrary for $x$ outside the leads and that $n_{o}-n_{i} \neq 0$ implies $\sigma_{a c}(U)=S^{1}$; also the spectral result remains true if condition (5) is only satisfied asymptotically in the sense of Corollary 3.5, point 3.

2. It is straightforward the extend the above results to hypersurfaces more general than $\Gamma$.

\section{Transport perpendicular to bulk boundaries, the Chalker-Coddington model}

This section is devoted to spectral and transport results for Chalker-Coddington models defined in the two-dimensional plane. It is an extension of the spectral analysis for the model in a quasi one-dimensional strip via a flux study lead in [4]. For background on the model we refer to [4] and references therein. Let

$$
\left\{S_{j, 2 k}\right\}_{j, k \in \mathbb{Z}}
$$

be a collection of unitary $2 \times 2$ matrices $S_{j, 2 k} \in U(2)$ called scattering matrices, even or odd according to the parity of $j$; they define the unitary operator which characterises the Chalker-Coddington model

$$
U: \ell^{2}\left(\mathbb{Z}^{2} ; \mathbb{C}\right) \rightarrow \ell^{2}\left(\mathbb{Z}^{2} ; \mathbb{C}\right)
$$


in the following way : denoting by $|j, k\rangle$ the canonical basis vectors of $l^{2}\left(\mathbb{Z}^{2} ; \mathbb{C}\right), U$ is defined according to figure (7) by:

$$
\begin{aligned}
& \left(\begin{array}{c}
U|2 j, 2 k\rangle \\
U|2 j+1,2 k-1\rangle
\end{array}\right):=S_{2 j, 2 k}\left(\begin{array}{c}
|2 j, 2 k-1\rangle \\
|2 j+1,2 k\rangle
\end{array}\right) \\
& \left(\begin{array}{c}
U|2 j+1,2 k\rangle \\
U|2 j+2,2 k+1\rangle
\end{array}\right):=S_{2 j+1,2 k}\left(\begin{array}{c}
|2 j+2,2 k\rangle \\
|2 j+1,2 k+1\rangle
\end{array}\right) .
\end{aligned}
$$

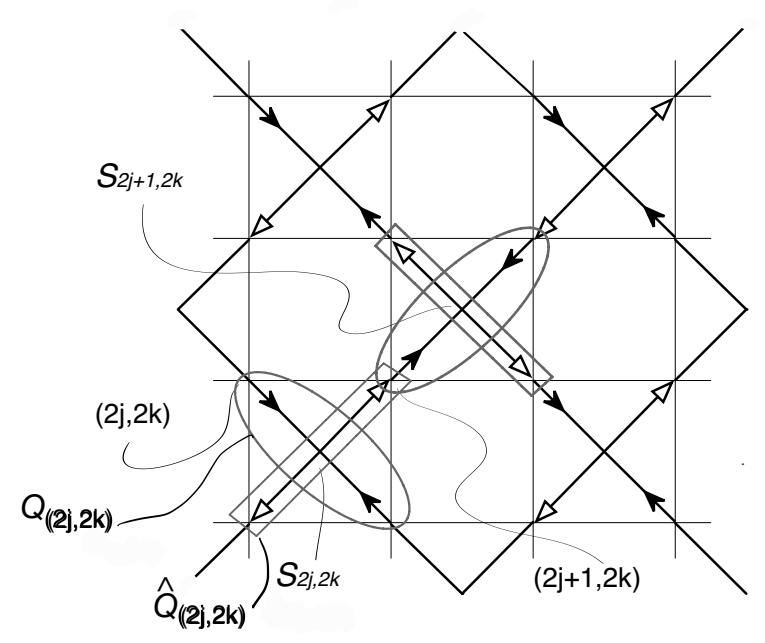

Figure 7: A Chalker-Coddington model with its incoming (solid arrows) and outgoing links.

The in- and outgoing subspaces of the scattering matrices play a prominent role in the following:

for $z=(j, 2 k) \in \mathbb{Z} \times 2 \mathbb{Z}$ denote $Q_{z}$ the orthogonal projections on

$$
\begin{aligned}
& \operatorname{Ran} Q_{2 j, 2 k}=\operatorname{span}\{|2 j, 2 k\rangle,|2 j+1,2 k-1\rangle\}, \\
& \operatorname{Ran} Q_{2 j+1,2 k}=\operatorname{span}\{|2 j+2,2 k+1\rangle,|2 j+1,2 k\rangle\} .
\end{aligned}
$$

and their images $\widehat{Q}_{z}=U Q_{z} U^{*}$ on

$$
\begin{aligned}
& \operatorname{Ran} \widehat{Q}_{2 j, 2 k}=\operatorname{span}\{|2 j+1,2 k\rangle,|2 j, 2 k-1\rangle\}, \\
& \operatorname{Ran} \widehat{Q}_{2 j+1,2 k}=\operatorname{span}\{|2 j+1,2 k+1\rangle,|2 j+2,2 k\rangle\} .
\end{aligned}
$$

Remarks 4.1. 1. In the original paper [11] the moduli of the elements of $S_{j, 2 k}$ were constant and the phases random variables. Here we have no restrictions. 
2. Note that $Q_{z}, \widehat{Q}_{z}$ do not depend on the collection of scattering matrices.

For the flux operator we observe the following :

Proposition 4.2. Let $G$ be a subset of $\mathbb{Z}^{2}$ and $P$ the multiplication operator $P=$ $\chi(x \in G)$,

$$
\Phi=U^{*} P U-P
$$

Then it holds

1. $\left[\Phi, Q_{z}\right]=0 \quad \forall z \in \mathbb{Z} \times 2 \mathbb{Z}$;

2. $\Phi Q_{z}=U^{*}\left(P \widehat{Q}_{z}\right) U-P Q_{z}$;

3. ind $(\Phi)$ is well defined iff for a $c, R>0: \sup _{|z|>R}\left\|\Phi Q_{z}\right\| \leq c<1$, then it holds

$$
\operatorname{ind}(\Phi)=\sum_{z,|z| \leq R} \operatorname{dim} \operatorname{Ran}\left(P \widehat{Q}_{z}\right)-\operatorname{dim} \operatorname{Ran}\left(P Q_{z}\right)
$$

Proof. By definition $Q_{z}, \widehat{Q}_{z}$ are multiplication operators on $\ell^{2}\left(\mathbb{Z}^{2} ; \mathbb{C}\right)$ thus commute with $P$, or

$$
\left[\Phi, Q_{z}\right]=\left[U^{*} P U, Q_{z}\right]=U^{*}\left[P, \widehat{Q}_{z}\right] U=0 .
$$

Also $\operatorname{ker}\left(\Phi^{2}-\mathbb{I}\right)=\bigoplus_{z \in \mathbb{Z} \times 2 \mathbb{Z}} \operatorname{ker}\left(\left(\Phi^{2}-\mathbb{I}\right) Q_{z}\right)$ and $\sigma\left(\Phi^{2}\right)=\bigcup_{z \in \mathbb{Z} \times 2 \mathbb{Z}} \sigma\left(\Phi^{2} Q_{z}\right)$. By assumption $\operatorname{dist}\left(\{1\}, \bigcup_{|z|>R} \sigma\left(\Phi^{2} Q_{z}\right)\right)>0$ thus 1 is an isolated finite dimensional eigenvalue and

$$
\begin{gathered}
\operatorname{ind}(\Phi)=\sum_{|z| \leq R} \operatorname{dim} \operatorname{ker}\left((\Phi-\mathbb{I}) Q_{z}\right)-\sum_{|z| \leq R} \operatorname{dim} \operatorname{ker}\left((\Phi+\mathbb{I}) Q_{z}\right)= \\
=\sum_{|z| \leq R} \operatorname{ind}\left(\Phi Q_{z}\right)=\sum_{|z| \leq R} \operatorname{trace}\left(\Phi Q_{z}\right)=\sum_{z,|z| \leq R} \operatorname{dim} \operatorname{Ran}\left(P \widehat{Q}_{z}\right)-\operatorname{dim} \operatorname{Ran}\left(P Q_{z}\right) .
\end{gathered}
$$

To define an appropriate projection $P$ we use some graph terminology. To the given $U$ be can associate the directed graph

$$
G=(V, E)
$$

with vertices in $V=\mathbb{Z}^{2}$ and whose directed edges are defined by

$$
\overrightarrow{x y} \in E \text { iff }\left\langle y, U_{c} x\right\rangle \neq 0
$$




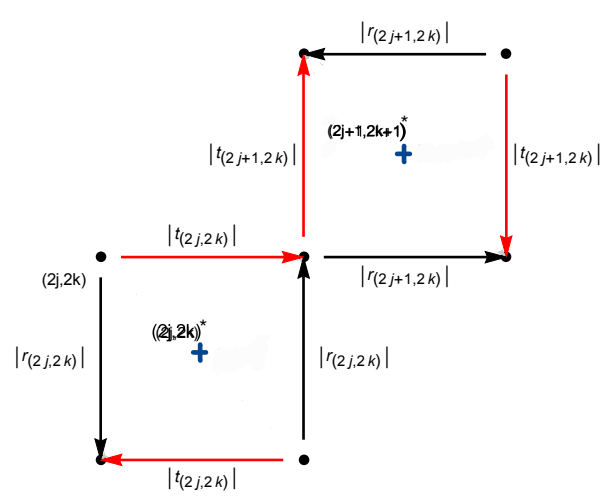

Figure 8: The weights of $G$.

where $U_{c}$ is the critical model which has all its scattering matrices equal to $\frac{1}{\sqrt{2}}\left(\begin{array}{cc}1 & -1 \\ 1 & 1\end{array}\right)$.

Some features of the given $U$ are then described by attributing

$$
\text { the weight }|\langle y, U x\rangle| \text { to the edge } \overrightarrow{x y} \in E
$$

and we call an edge open of its weight does not vanish, see figure (8).

The dual graph

$$
G^{*}=\left(V^{*}, E^{*}\right)
$$

has its vertices at the center of the faces of $G$

$$
V^{*}=\mathbb{Z}^{2}+\frac{1}{2}(1,-1)
$$

and its edges (which we call links to distinguish) connect all neighbours at distance 1 thus bisecting the edges of $G$. We use the notations:

$$
V^{*} \ni v^{*}=\left(x_{1}^{*}, x_{2}^{*}\right):=\left(x_{1}, x_{2}\right)+\frac{1}{2}(1,-1)
$$

for integers $x_{1}, x_{2}$ and say that

$$
x_{j}^{*} \in \text { Even if } x_{j} \in 2 \mathbb{Z}, \quad x_{j}^{*} \in O d d \text { if } x_{j} \in 2 \mathbb{Z}+1 .
$$

Our parameters for the scattering matrices $S_{z}, z \in \mathbb{Z} \times 2 \mathbb{Z}$ are:

$$
S_{z}=q_{z}\left(\begin{array}{cc}
r_{z} & -t_{z} \\
\overline{t_{z}} & \overline{r_{z}}
\end{array}\right) \quad \text { with } q_{z} \in S^{1}, r_{z}, t_{z} \in \mathbb{C} \text { s.t. }\left|r_{z}\right|^{2}+\left|t_{z}\right|^{2}=1 \text {. }
$$

Now consider a partition of $G$ in two infinite connected subgraphs $G_{+}$and $G_{-}$and consider a path in $G^{*}$ which bisects the edge boundary of $G_{+}$. 


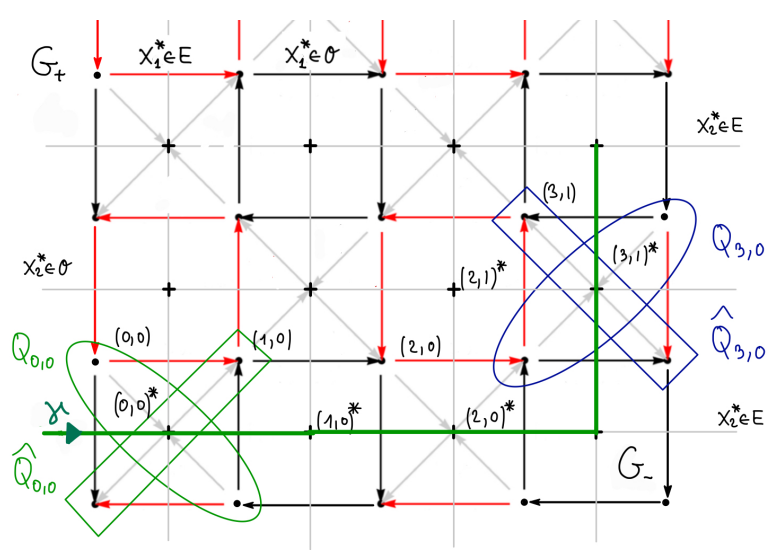

Figure 9: Edges of weight $|r|$ (black) are crossed by links on $\gamma$ incident to $\left(x_{1}^{*}, x_{2}^{*}\right)$ which are : horizontal if $x_{2}^{*} \in$ Even or vertical if $x_{1}^{*} \in O d d$. Red edges are of weight $|t|$.

To be specific apprehend such a path as a continuously parametrized injective piecewise unit speed curve of segments of length one

$$
\gamma: \mathbb{R} \rightarrow G^{*} \subset \mathbb{R}^{2} \text { such that } \gamma(t) \in V^{*} \text { and } \overrightarrow{\gamma(t) \gamma(t+1)} \in E^{*} \text { for } t \in \mathbb{Z}
$$

oriented such that $G_{+}$is to the left of $\gamma$; we call it an admissible path. Let $V_{+}$be the set of vertices of $G_{+}$and consider its projection and flux operator in $\ell^{2}\left(\mathbb{Z}^{2} ; \mathbb{C}\right)$ :

$$
P_{\gamma}=\chi\left(x \in V_{+}\right), \quad \Phi_{\gamma}=U^{*} P_{\gamma} U-P_{\gamma} .
$$

In the following we shall give sufficient conditions for non-trivial $\operatorname{ind}\left(\Phi_{\gamma}\right)$. By the general theory of Theorem 2.1 and Proposition 2.2 we know that all vertices which are not incident to the edge belong to $\operatorname{ker}\left(\Phi_{\gamma}\right)$; in view of proposition 4.2 we label the set of interesting vertices as follows:

Definition 4.3. Let $\gamma: \mathbb{R} \rightarrow G^{*}$ be an admissible path. We call $E_{\gamma} \subset E$ the set of edges bisected by $\gamma$ and $V_{\gamma}$ the labels of subspaces $\operatorname{Ran} Q_{z}$ which contain vertices incident to $E_{\gamma}$ :

$$
V_{\gamma}:=\left\{z \in \mathbb{Z} \times 2 \mathbb{Z} ; P_{\gamma}^{\perp} U_{c} P_{\gamma} Q_{z} \neq 0 \text { or } P_{\gamma} U_{c} P_{\gamma}^{\perp} Q_{z} \neq 0\right\} .
$$

$\gamma$ is called an $r$-path in $I \subset \mathbb{R}$ if its restriction to $I$ bisects only black edges of weight $\left|r_{z}\right|$ for $z \in V_{\gamma}$. A t-path in $I$ is defined analogously.

Remark 4.4. Observe that according to figure(9) any link of an $r$-path can be incident to dual vertices in Odd $\times$ Even but it has to be horizontal at Even $\times$ Even and vertical at $O d d \times O d d$. For a $t$-path the opposite is true. 
Lemma 4.5. Let $\gamma$ be an admissible $r$-path in $\mathbb{R}$; then it holds for the operator and the trace norm:

$$
\left\|\Phi_{\gamma}\right\| \leq \sup _{z \in V_{\gamma}}\left|r_{z}\right|, \quad\left\|\Phi_{\gamma}\right\|_{1} \leq \text { const. } \sum_{z \in V_{\gamma}}\left|r_{z}\right| .
$$

Proof. We suppress the subscript $\gamma$ to $\Phi$ and $P$ for the proof. Only states with label in $V_{\gamma}$ contribute and $\left[\Phi^{2}, Q_{z}\right]=0$ so we have

$$
\Phi^{2}=P U^{*} P^{\perp} U P+P^{\perp} U^{*} P U P^{\perp}=\sum_{z \in \mathbb{Z} \times 2 \mathbb{Z}} \Phi^{2} Q_{z}=\sum_{z \in V_{\gamma}} \Phi^{2} Q_{z}=\sum_{z \in V_{\gamma}}\left|r_{z}\right|^{2} Q_{z} .
$$

Now $\left[\Phi^{2}, P\right]=0$ and $\gamma$ crosses $Q_{z}$ horizontally or vertically and only edges of weight $\left|r_{z}\right|$ thus $\Phi^{2} Q_{z}=\left|r_{z}\right|^{2} Q_{z}$, see figure (10). It follows that $\left|\Phi_{\gamma}\right|=\sum_{z \in V_{\gamma}}\left|r_{z}\right| Q_{z}$ which implies the assertion.

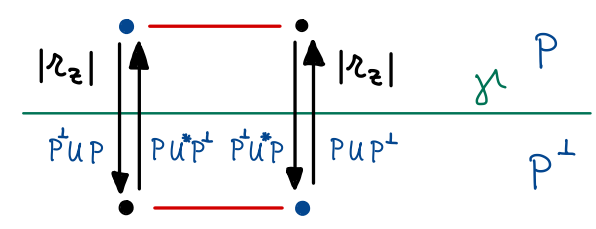

Figure 10: The action of $\Phi^{2} Q_{z}$ for an $r$-path

Proposition 4.6. Let $\gamma$ be an admissible $r$-path in $I=\mathbb{R} \backslash[-N, N]$ for an $N>1$ and such that for a $c>0$

$$
\left|r_{z}\right| \leq c<1 \quad \forall z \in V_{\gamma \mid I}
$$

then $\operatorname{ind}\left(\Phi_{\gamma}\right)$ is well defined and it holds

$$
\operatorname{ind}\left(\Phi_{\gamma}\right)=0 .
$$

The analogous statement holds true for a t-path.

Proof. We suppress the subscript $\gamma$ to $\Phi$ and $P$ for the proof. It follows from Proposition (4.2) and Lemma (4.5) that $\operatorname{ind}(\Phi)$ is well defined because

$$
\sum_{z \in V_{\gamma}} \Phi Q_{z}-\sum_{z \in V_{\gamma \mid I}} \Phi Q_{z}
$$

is of finite rank and

$$
\operatorname{ind}(\Phi)=\sum_{z \in V_{\gamma[[-N, N]}} \operatorname{dim} \operatorname{Ran}\left(P \widehat{Q}_{z}\right)-\operatorname{dim} \operatorname{Ran}\left(P Q_{z}\right) .
$$


If $\gamma$ is already an $r$-path in all $\mathbb{R}$ then $\operatorname{dim} \operatorname{Ran}\left(P \widehat{Q}_{z}\right)=\operatorname{dim} \operatorname{Ran}\left(P Q_{z}\right)=1, \forall z \in$ $V_{\gamma}$ because only $r$ edges are bisected thus $P$ splits $\operatorname{Ran} Q_{z}$ and $\operatorname{Ran} \widehat{Q}_{z}$ horizontally or vertically according parity, c.f. (4.4). Thus ind $\left(\Phi_{\gamma}\right)=0$.

If not, choose two integers $N_{ \pm} \in \mathbb{Z}$ such that $\pm N_{ \pm}> \pm N$ and such that $\gamma\left(N_{ \pm}\right) \in$ $O d d \times$ Even thus all links incident to the dual vertices $\gamma\left(N_{ \pm}\right)$bisect edges of weight $|r|$. Then we can define a new admissible path $\widehat{\gamma}$ replacing the part $\gamma \uparrow_{\left(-N_{-}, N_{+}\right)}$by an $r$ path inside $G_{+}$connecting $\gamma\left(N_{-}\right)$to $\gamma\left(N_{+}\right)$. The difference $P_{\gamma}-P_{\widehat{\gamma}}$ is of finite rank thus

$$
\operatorname{ind}\left(\Phi_{\gamma}\right)=\operatorname{ind}\left(\Phi_{\widehat{\gamma}}\right)=0
$$

Theorem 4.7. Let $U$ be a Chalker Coddington model such that there exists an admissible path $\gamma$ which is an $r$-path in $(-\infty,-N]$ and a $t$-path in $[N, \infty)$ for an integer $N>1$, and such that for a $c>0$

$$
\left|r_{z}\right| \leq c<1 \quad \forall z \in V_{\gamma\lceil(-\infty,-N]} \text { and }\left|t_{z}\right| \leq c<1 \quad \forall z \in V_{\gamma\lceil[N, \infty)} .
$$

Then $\operatorname{ind}\left(\Phi_{\gamma}\right)$ is well defined and it holds

$$
\left|\operatorname{ind}\left(\Phi_{\gamma}\right)\right|=1
$$

In addition

1. $\Phi_{\gamma}$ is compact iff $\lim _{s \rightarrow-\infty} r_{\gamma(s)}=0=\lim _{s \rightarrow \infty} t_{\gamma(s)}$ and then $\sigma(U)=S^{1}$

2. $\Phi_{\gamma}$ is trace class iff $\sum_{z \in V_{\gamma \uparrow(-\infty,-N]}}\left|r_{z}\right|+\sum_{z \in V_{\gamma \uparrow[N, \infty)}}\left|t_{z}\right|<\infty$ and then

$$
\sigma_{a c}(U)=S^{1}
$$

and a trace class pertubation of $U$ contains a shift operator.

Proof. If $\gamma$ is a path which switches from $r$ to $t$ on one site, i.e : if $\gamma$ is an $r$-path in $(-\infty, 0)$ and a $t$-path in $[0, \infty)$, then as a corollary of Propositions 4.2 and 4.6 we have

$$
\operatorname{ind}(\Phi)=\sum_{\left.z \in V_{\gamma \mid}-N, N\right]} \operatorname{dim} \operatorname{Ran}\left(P \widehat{Q}_{z}\right)-\operatorname{dim} \operatorname{Ran}\left(P Q_{z}\right)=\operatorname{dim} \operatorname{Ran}\left(P \widehat{Q}_{z_{0}}\right)-\operatorname{dim} \operatorname{Ran}\left(P Q_{z_{0}}\right)
$$

where $z_{0}$ is such that $\gamma(0)$ is the center of the face defined by the vertices in $\operatorname{Ran} \widehat{Q}_{z_{0}}$ and $\operatorname{Ran} Q_{z_{0}}$. By the symmetry of the problem these two edges are either incoming or outgoing to $G_{+}$thus $\operatorname{dim} \operatorname{Ran} P Q_{z_{0}}=1$ and $\operatorname{dim} \operatorname{Ran} P \widehat{Q}_{z_{0}}=0$ or the other way round, see figure (11). Thus $\left|\operatorname{ind}\left(\Phi_{\gamma}\right)\right|=1$. 


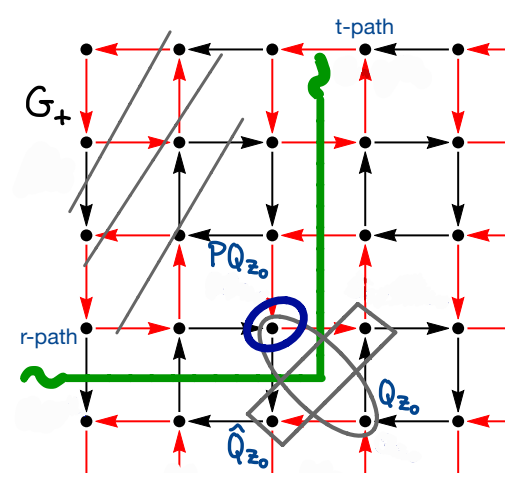

Figure 11: Crossover from $r$ to $t$.

If not, choose two integers $N_{ \pm} \in \mathbb{Z}$ such that $\pm N_{ \pm}> \pm N$ and such that $\gamma\left(N_{-}\right) \in$ Odd $\times$ Even and $\gamma\left(N_{+}\right) \in$ Even $\times$ Odd thus all links incident to the dual vertices $\gamma\left(N_{-}\right)$bisect edges of weight $|r|$ and all links incident to the dual vertices $\gamma\left(N_{+}\right)$ bisect edges of weight $|t|$. Then we can define a new admissible path $\widehat{\gamma}$ replacing the part $\gamma \uparrow_{\left(-N_{-}, N_{+}\right)}$by an $r$ path inside $G_{+}$connecting $\gamma\left(N_{-}\right)$to $\gamma\left(N_{+}\right)$, c.f. figure (12). The difference $P_{\gamma}-P_{\widehat{\gamma}}$ is of finite rank thus

$$
\operatorname{ind}\left(\Phi_{\gamma}\right)=\operatorname{ind}\left(\Phi_{\widehat{\gamma}}\right)
$$

The additional assertions follow from lemma 4.5 and theorem 2.1.

As by unitarity it holds $\left|r_{z}\right|^{2}+\left|t_{z}\right|^{2}=1$ it follows:

Corollary 4.8. The critical Chalker Coddington model defined by $\left|r_{z}\right|=\left|t_{z}\right|=\frac{1}{\sqrt{2}}, \forall z$ admits a projection $P$ with non-trivial index

$$
\operatorname{ind}(\Phi) \neq 0
$$

for any distribution of phases of its scattering matrices.

More generally: a Chalker Coddington model defined by a collection of scattering matrices such that for $c_{1}, c_{2} \in(0,1)$ and all $z$ : $0<c_{1}<\left|r_{z}\right|<c_{2}<1$, admits a projection with non trivial index.

\section{Spectral implications and anomalous charge trans- port}

Observe that Corollary 4.8 applies to the dynamically localised case studied in $[2,1]$, i.e.: $\left|r_{z}\right|=$ const $\neq 0$ sufficiently small and i.i.d. and uniformly distributed phases; another 


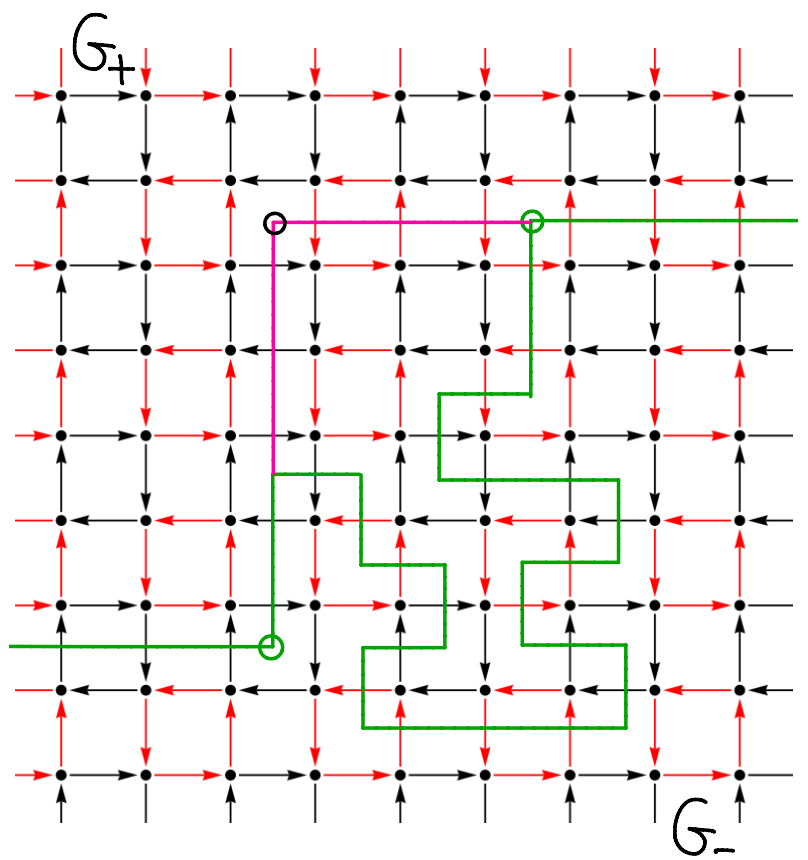

Figure 12: Join $r$ path to $t$ path.

remarkable situation which is covered by the Corollary is the translation invariant $0<$ $\left|r_{z}\right|=$ const $\neq \frac{1}{\sqrt{2}}$ case with all phases equal to unity. In the first case the system is almost surely dynamically localised, in the second case the spectrum is absolutely continuous and exhibits gaps. In both situations, as in the critical case $\left|r_{z}\right|=\frac{1}{\sqrt{2}}$, the flux operator $\Phi_{\gamma}$ is not trace class or even compact.

To close this analysis of the transport properties of the Chalker-Coddington model, we argue that the random case is an example of a non trivial anomalous charge transport in a regime of Anderson localisation in the following sense:

consider $U$ defined by $\left\{S_{z}\right\}_{z \in \mathbb{Z} \times 2 \mathbb{Z}}$ such that $\left|r_{z}\right|=$ const $\neq 0$ and such that $\sigma(U)$ is pure point. For $\varepsilon_{0}>0$ choose an admissible path $\gamma$ like in Theorem 4.7 which is an $r$-path in $\left(-\infty,-\frac{1}{\varepsilon_{0}}\right]$ and a $t$-path in $\left[\frac{1}{\varepsilon_{0}}, \infty\right)$. Now define for $0<\varepsilon \leq \varepsilon_{0}$ the unitary $U_{\varepsilon}$ by the scattering matrices

$$
S_{z}^{\varepsilon}:=\left\{\begin{array}{cl}
\left(\begin{array}{cc}
0 & -1 \\
1 & 0
\end{array}\right) & z \in V_{\gamma \uparrow\left(-\infty,-\frac{1}{\varepsilon}\right]} \\
\left(\begin{array}{cc}
1 & 0 \\
0 & 1
\end{array}\right) & z \in V_{\gamma \uparrow\left(\frac{1}{\varepsilon}, \infty\right]} \\
S_{z} & z \text { elsewhere }
\end{array}\right.
$$

Let $\Phi:=U^{*} P_{\gamma} U-P_{\gamma}, \Phi_{\varepsilon}:=U_{\varepsilon}^{*} P_{\gamma} U_{\varepsilon}-P_{\gamma} . \Phi_{\varepsilon}$ is trace class, thus, considered as a symbol, its fermionic second quantisation is a bona fide operator in the $C A R$ 
observables. Furthermore for the full quasifree state, whose symbol is the identity operator $\mathbb{I}$, the mean transported charge across $\gamma$ reads: $\operatorname{trace}\left(\mathbb{I} \Phi_{\varepsilon}\right)=\operatorname{trace}\left(\Phi_{\varepsilon}\right)=$ $\operatorname{ind}\left(\Phi_{\varepsilon}\right)$. From Theorem (4.7) we know that $\left|\operatorname{ind}\left(\Phi_{\varepsilon}\right)\right|=1$ so that

$$
\lim _{\varepsilon \rightarrow 0} \operatorname{trace}\left(\mathbb{I} \Phi_{\varepsilon}\right)=\operatorname{ind}(\Phi) \neq 0
$$

and we have an anomalous charge transport by $U$ across $\gamma$. Note that $U_{\varepsilon} \rightarrow U$ and $\Phi_{\varepsilon} \rightarrow \Phi$ strongly.

We draw an analogy between this very concrete example and the phenomenon of anomalous currents in gauge field theories, see [20].

\section{Acknowledgments}

We thank Claude Alain Pillet for insightful discussions. We acknowledge the warm hospitality and support of the Centre de Recherches Mathématiques in Montréal: A.J. was a CRM-Simons Scholar-in-Residence Program, and J.A. was a guest of the Thematic Semester Mathematical Challenges in Many Body Physics and Quantum Information.

\section{References}

[1] Asch, J., Bourget, O., Joye, A., Localization Properties of the ChalkerCoddington Model. Ann. H. Poincaré, 11, 1341-1373, (2010).

[2] Asch, J., Bourget, O., Joye, A., Dynamical Localization of the ChalkerCoddington Model far from Transition, J. Stat. Phys., 147, 194-205 (2012).

[3] Asch, J., Bourget, O., Joye, A., Spectral Stability of Unitary Network Models, Rev. Math. Phys., 27, 1530004, (2015).

[4] Asch, J., Bourget, O., Joye, A., Chirality induced Interface Currents in the Chalker Coddington Model, J. Spectr. Theory 9 (2019), no. 4, 1405-1429.

[5] Asch, J., Joye, A., "Lower Bounds on the Localisation Length of Balanced Random Quantum Walks", Letters in Mathematical Physics,109, 21332155 (2019)

[6] Ahlbrecht, A., Scholz, V.B., Werner, A.H.: Disordered quantum walks in one lattice dimension, J. Math. Phys. 52, 102201 (2011)

[7] Avron, J., Seiler, R., Simon, B., The Index of a Pair of Projections, J. Func. Anal., 120, 220-237, (1994) 
[8] Avron, J., Seiler, R., Simon, B., Charge deficiency, charge transport and comparison of dimensions, Comm. Math. Phys., 159, 399-4227, (1994)

[9] Bourget, O., Howland, J., Joye, A. Spectral analysis of unitary band matrices. Commun. Math. Phys., 234, 191-227, (2003).

[10] Cedzich, C., Geib, T., Grünbaum, F. A., Stahl, C., Velázquez, L., Werner, A. H., Werner, R. F., The topological classification of one-dimensional symmetric quantum walks. Ann. H. Poincaré, 19, 325-383, (2018)

[11] Chalker, J. T., Coddington, P. D. Percolation, quantum tunnelling and the integer Hall effect. J. Phys. C: Solid State Physics, 21, 2665, (1988).

[12] Delplace, P., Fruchart, M., Tauber, C. Phase rotation symmetry and the topology of oriented scattering networks. Phys. Rev. B, 95, 205413, (2017).

[13] Graf, G. M., Tauber, C. Bulk-Edge correspondence for two-dimensional Floquet topological insulators. Ann. H. Poincaré, 19, 709-741, (2018)

[14] E.Hamza, A.Joye : Spectral Transition for Random Quantum Walks on Trees, Commun. Math. Phys., 326, 415-439, (2014).

[15] A.Joye, L.Marin : "Spectral Properties of Quantum Walks on Rooted Binary Trees", J. Stat. Phys., 155, 1249-1270, (2014).

[16] A.Joye and M.Merkli: "Dynamical Localization of Quantum Walks in Random Environments", J. Stat. Phys., 140, 1025-1053, (2010).

[17] A.Joye: "Dynamical Localization for $d$-Dimensional Random Quantum Walks", Quantum Inf. Proc., Special Issue: Quantum Walks, 11, 12511269, (2012).

[18] Kitaev, A. Anyons in an exactly solved model and beyond. Ann. of Phys., 321, 2-111, (2006).

[19] Kramer, B., Ohtsuki, T., Kettemann, S., Random network models and quantum phase transitions in two dimensions, Physics Reports, 417, 211, (2005).

[20] Nakahara, M. Geometry, Topology and Physics, chapter 13.2 (IOP, Briston and Philadelphia, 2003)

[21] R. Portugal, Quantum Walks and Search Algorithms (Springer, New York, 2013). 
[22] Rudner M.S, Lindner N. H., Berg E., Levin M., "Anomalous edge states and the bulk-edge correspondence for periodically driven two-dimensional systems". Phys. Rev. X 3, 031005 (2013).

[23] Sz.-Nagy, B., Foias, C., Berkovici, H., Kérchy, J., Harmonic Analysis of Operators in Hilbert Spaces, Springer (2010).

[24] Sadel, C., Schulz-Baldes, H. Topological boundary invariants for Floquet systems and quantum walks. Math Phys Anal Geom (2017) 20-22.

[25] Venegas-Andraca, Salvador Elias, Quantum walks: a comprehensive review, Quantum Inf. Process., 11, 1015-1106, (2012). 